Z. Epileptol. 2020 33:238-239

https://doi.org/10.1007/s10309-020-00339-3

Online publiziert: 15. Juni 2020

(c) Springer Medizin Verlag GmbH, ein Teil von Springer Nature 2020

Frank Brandhoff' · Thomas Mayer' · Günter Krämer ${ }^{2}$

${ }^{1}$ Kleinwachau - Sächsisches Epilepsiezentrum Radeberg, Radeberg, Deutschland

${ }^{2}$ Neurozentrum Bellevue, Zürich, Schweiz

\title{
Nachruf auf Professor Dr. med. habil. Detlef Müller (1926-2020)
}

Psychotherapie ergänzte. Im Jahr 1959 wurde er zum Oberarzt und Stellvertreter des 1. Oberarztes der Neurologisch-Psychiatrischen Klinik der Universität Leipzig ernannt. Noch vor dem Mauerbau (1960) hielt er sich zu einem Studienaufenthalt in Zürich bei dem klinischen Neurophysiologen Rudolf Hess (1913-2007) auf, 1962 in Warschau bei dem Neurologen Jerzy Majkowski (1928-2019) [2].

Im Jahr 1965 wechselte Detlef Müller als Oberarzt an die Medizinische Akademie "Carl Gustav Carus“ in Dresden (= das heutige Universitätsklinikum). 1968 habilitierte er sich mit einer Arbeit über den Einfluss des Karotisdruckversuchs auf das EEG (1972 als Monographie publiziert [3]) und erlangte 1970 die Lehrbefähigung („Facultas Docendi“) für Neurologie und Psychiatrie. Im gleichen Jahr wurde er zum Leiter der EEGAbteilung und der Epilepsieambulanz ernannt. Im Jahr 1975 erhielt er zusätzlich einen Lehrauftrag für „Psychopathologie“ bzw. „Klinisch-psychologische Grundlagen“ an der Technischen Universität Dresden. Im Februar 1979 erfolgte die Ernennung zum Hochschuldozenten für Neurologie/Psychiatrie, und nach jahrelanger Nichtberücksichtigung erst am 15.09.1990 - also 2 Wochen vor der Wiedervereinigung mit der Bundesrepublik Deutschland und kurz vor seinem altersbedingten Ausscheiden im darauffolgenden Jahr - zum außerordentlichen Professor für Neurologie an der Medizinischen Akademie Dresden [1].

Als Epilepsie-relevante Leistungen von Detlef Müller sind u. a. seine Initiierung der Gründung der DDR-Sektion der ILAE und die Tätigkeit als deren Sekretär von 1980 bis 1987 sowie die auch nach seiner Pensionierung bis 2009 fortgeführte Organisation und Leitung von Fort- und Weiterbildungsveranstaltungen zur klinischen Elektroenzephalographie und zu Anfallserkrankungen hervorzuheben. Als Auszeichnungen seien die „Hans-Berger-Medaille“ der Gesellschaft für Neuro-Elektrodiagnostik der DDR 1976 und die Ehrenmedaille der Medizinischen Akademie „Carl Gustav Carus“ in Dresden 1989 erwähnt. Er war auch Autor zahlreicher Artikel (Auswahl: [4-9]) und - zusammen mit seiner nicht mit ihm verwandten Namensvetterin Jutta Müller $\left({ }^{*} 1934\right)$ - einer Monographie über Lachen als epileptische Manifestation [10].

In Dresden und im Epilepsiezentrum Kleinwachau fehlte er bei keiner Fortbildung und war ein aktiver, ja gefürchteter Diskutant. Gefürchtet waren auch seine oft langen Briefe, die er Referenten nach ihren Vorträgen schickte, um Sachverhalte „bis zu Ende“ zu diskutieren. Besonders lebhaft wurde es bei der Differenzialdiagnose von epileptischen Anfällen oder Synkopen; in aller Regel war er nie ganz zufriedenzustellen, weil seine Fragen nicht genau genug beantwortet wurden. Er selbst meinte dazu, dass er es „mit den Worten" sehr genau nehme, also wie etwas formuliert wurde. Dies galt auch für seine Leserbriefe zu Publikationen [8, 9].

Detlef Müller hatte 1973 den frühen Tod seines Zwillingsbruders und 1997 den seiner Ehefrau nach langer Krankheit zu betrauern. Vielleicht war es das Vermächtnis seiner Frau, das er mit seinem sozialen Engagement erfüllte. Er hatte nämlich noch eine weitere, weitgehend unbekannte Seite außerhalb der Medizin. Seit 1997 engagierte er sich 


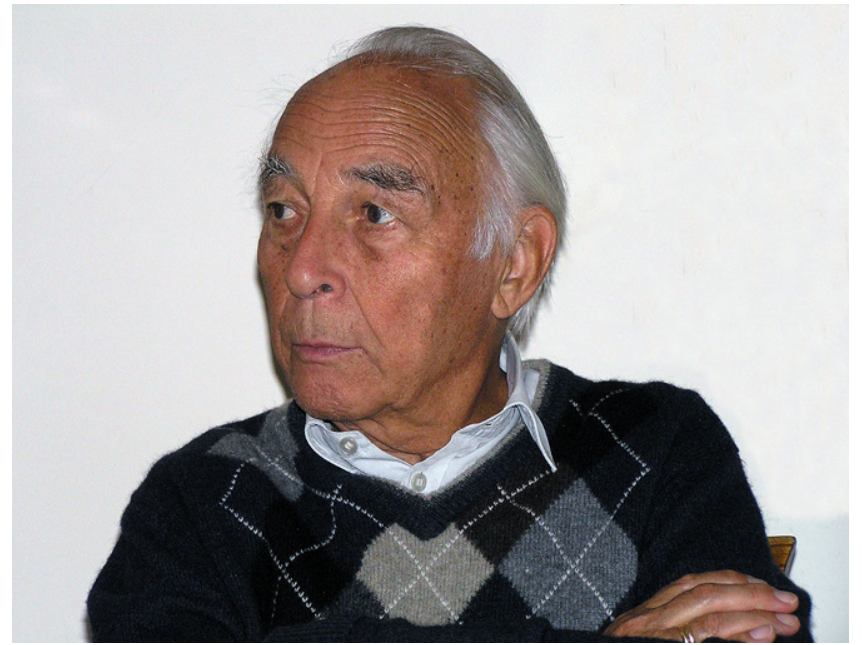

Abb. $1<$ Prof. Dr. med. habil. Detlef Müller
9. Müller D (2011) Ein weiterer Offener Brief zum Problemfeld Anfallsdifferenzialdiagnostik (Leserbrief).ZEpileptol 24:224

10. Müller D (2017) Notwendige Anmerkungen zur jetzigen Begrifflichkeit der akuten organischen Psychosyndrome. Fortschr Neurol Psychiatr 85:135

11. Müller D, Müller J (1980) Lachen als epileptische Manifestation. Sammlung zwangloser Abhandlungen aus dem Gebiete der Psychiatrie und Neurologie, Bd. 48. VEB G. Fischer, Jena mit vielen Nachtdiensten zur Betreuung von Obdachlosen oder dem Ausliefern von Essen auf Rädern für Bedürftige beim ökumenischen WohnungslosenNachtcafé in Dresden oder begleitete in der Flüchtlingshilfe Kranke vor allem bei Arztbesuchen und baute so Brücken. Dass er ausdrücklich nie an einem Industrie-gesponserten Essen teilnahm oder sich auch nur einen Kugelschreiber schenken ließ, ist gemessen daran schon nur noch Anekdote.

Als wir ihm letztes Jahr zu seinem 93. Geburtstag aus Kleinwachau eine Karte und ein Buch schenkten, in dem er selbst sehr rührend erwähnt wurde, rief er an und schilderte, dass es ihm nicht gut gehe, er weder gut laufen noch gut sehen könne und die besagte Stelle im Buch nicht gefunden habe. Aber er war auch mit 93 noch ganz der alte „Nervenmüller“, als den er sich manchmal halb scherzhaft selbst bezeichnete. Dass dies auch seine Berechtigung hatte, können viele bestätigen, die häufiger Kontakt mit ihm hatten, z. B. als federführender Herausgeber einer Fachzeitschrift und einer nur mit großer Mühe zu erzielenden Akzeptanz von kritischen Kommentaren oder gar Ablehnungen eingereichter Manuskripte.

Wir erinnern Detlef Müller als einen der wenigen Neurologen und Epileptologen der früheren DDR, der nach der Wiedervereinigung nicht verstummte. Wir vermissen seinen feinen Humor, der nie verletzend war. Was bleibt, ist unsere Einsicht, dass fachliche Streitlust eben die höchste Technik des kollegialen Austau- sches und vielleicht auch nur eine besondere Ausdrucksform kollegialer Nähe ist. Unser Mitgefühl gilt seinen Kindern und Enkelkindern.

\section{Korrespondenzadresse}

\section{Dr. med. Günter Krämer}

Neurozentrum Bellevue

Theaterstr. 8, 8001 Zürich, Schweiz

g.kraemer@epilepsie-med.de

\section{Literatur}

1. Heidel C-P, Lienert M (2005) Die Professoren der Medizinischen Fakultät Carl Gustav Carus Dresden und ihrer Vorgängereinrichtungen 1814-2004. K. G. Saur, München, S 157-158 (im Auftrag des Dekans der medizinischen Fakultät Carl Gustav Carus und des Vorstands des Universitätsklinikums Carl Gustav Carus an der Technischen Universität Dresden)

2. Krämer G (2012) Lexikon der Epileptologie, 2. Aufl. Hippocampus, Bad Honnef, S 921

3. Müller D (1972) Der Karotisdruckversuch als Provokationsmethode in der klinischen Elektroenzephalographie Bd. 42.VEB G. Fischer, Jena

4. Müller D (1980) On the problem of nonepileptic drop attacks. In: Majkowski J (Hrsg) Epilepsy. A clinical and experimental research Proceedings of the Second European Regional Conference on Epilepsy, Warsaw, 05-07.10.1978. Monographs in neural sciences, Bd. 5. S. Karger, Basel, S298-300

5. Müller D (1988) Probleme der Differentialdiagnostikzerebraler Anfälle. Z Arztl Fortbild 82:989-992

6. Müller D (1989) Differentialdiagnostik zerebraler Anfälle mit Stürzen. ZKlin Med 44:1699-1702

7. Müller D (1993) Anfallsweise Zustände von Angst und Erregung am Beispiel des sympathikotonen Anfalls. In: Lange E (Hrsg) Angst und Erregung Schmerz und Verstimmung. J. A. Barth, Leipzig, Berlin, Heidelberg, S53-60

8. Müller D (2011) Offener Brief zur DoppelDVD "Epilepsie leben - Epilepsie verstehen" (Leserbrief).ZEpileptol 24:139-140 